\title{
Association between interleukin-33 and ovarian cancer
}

\author{
XIAOQING LIU ${ }^{1,2^{*}}$, DWAYNE M. HANSEN ${ }^{3 *}$, NOAH J. TIMKO ${ }^{2 *}$, ZIWEN ZHU $^{2}$, ANDREW AMES $^{3}$, \\ CHENGLU QIN $^{2}$, MICHAEL B. NICHOLL ${ }^{2}$, QIAN BAI ${ }^{2}$, XUHUI CHEN $^{3}$, \\ MARK R. WAKEFIELD ${ }^{2}$, GAGE WEST ${ }^{2}$ and YUJIANG FANG ${ }^{2,3}$ \\ ${ }^{1}$ Department of Anatomy, Anhui College of Traditional Chinese Medicine, Wuhu, Anhui 241000, P.R. China; \\ ${ }^{2}$ Department of Surgery, University of Missouri School of Medicine, Columbia, MO 65212; \\ ${ }^{3}$ Department of Microbiology, Immunology and Pathology, Des Moines University \\ College of Osteopathic Medicine, Des Moines, IA 50312, USA
}

Received December 15, 2017; Accepted November 13, 2018

DOI: $10.3892 /$ or.2018.6918

\begin{abstract}
Ovarian cancer is the leading cause of cancerassociated mortality in the female reproductive system. Interleukin (IL)-33 and its receptor IL 1 receptor like 1 (also termed ST2) are expressed by many cell types including epithelial cells. The role of IL-33 in the pathogenesis of neoplasia remains controversial. The authors previously demonstrated that IL-33 inhibits the growth of pancreatic cancer cells. The present study was performed to explore if IL-33 has any direct effects on ovarian cancer cells. A clonogenic survival assay, immunohistochemistry (IHC), proliferation kit and caspase-3 activity kit were all used to evaluate the direct effects of IL-33 on cell proliferation and apoptosis of a widely studied ovarian cancer cell line, A2780. The possible molecular mechanisms were further evaluated with reverse transcription-polymerase chain reaction and IHC. It was demonstrated that the percentage of colonies and the optical density value of cancer cells were all increased in the presence of IL-33; however, the relative caspase- 3 activity in cancer cells was decreased in the presence of IL-33. Molecular mechanism studies revealed that the pro-proliferative effect of IL-33 on cancer cells was associated with decreased levels of p27, and the anti-apoptotic effect of IL-33 was associated with levels of Fas cell surface death receptor (Fas) and tumor necrosis factor-related apoptosis-inducing ligand receptor 1 (TRAILR1). Therefore, IL-33 promoted proliferation and inhibited apoptosis of ovarian cancer cells by downregulation of p27, Fas and TRAILR1. Contrary to previous studies demonstrating an anti-tumor
\end{abstract}

Correspondence to: Dr Yujiang Fang, Department of Microbiology, Immunology and Pathology, Des Moines University College of Osteopathic Medicine, Des Moines, IA 50312, USA

E-mail: yujiang.fang@dmu.edu

${ }^{*}$ Contributed equally

Key words: interleukin-33, apoptosis, proliferation, epithelial, ovary, ovarian cancer effort in pancreatic cancer, the results of the present study indicated that IL-33 exhibited a significant onco-promoting effect on ovarian cancer. Accordingly, the inhibition of IL-33 may be a promising therapeutic strategy for ovarian cancer.

\section{Introduction}

Ovarian cancer is regarded as one of the most dangerous gynecological malignancies in the United States, as a consequence to its insidious onset, late presentation, and limited advances in therapy (1-3). As of 2016, a woman living in the United States carries a 1 in 75 lifetime risk of the disease, as well as a 1 in 100 risk of associated death; a statistic which over the last 40 years, has only changed by small increments, while other cancers have experienced large breakthroughs both in screening and in treatment $(4,5)$.

Interleukin (IL)-33 was identified in 2005. Its associated signaling, which occurs through the receptor IL-1 receptor like 1 (ST2), was revealed to activate mitogen activated protein kinase and nuclear factor (NF)- $\kappa \mathrm{B}$ responses, eventually leading to an in vitro $\mathrm{T}$ helper cell 2 polarized type response (6,7). Mechanistic investigation of IL-33 and its role in carcinogenesis has only recently begun (6). Various studies have indicated that IL-33 has a pro-tumor effect in cholangiocarcinoma, colon and breast cancer (8-10). IL-33 has been identified as a useful biomarker in the diagnosis or prediction of prognosis in non-small cell lung cancer (11). Interestingly, other studies have suggested that IL-33 has an anti-tumor effect in colon cancer $(12,13)$ and our previous study on pancreatic and colon cancer echoes these findings (14). This suggests the complex role of IL-33 in the pathogenesis of neoplasia. In ovarian cancer, increased levels of IL-33 and ST2 have been found in neoplastic lesions (15). However, the direct effects of IL-33 on ovarian cancer are still unknown, therefore the present study addressed the lack of research through the use of a widely studied ovarian cancer cell line, A2780.

\section{Materials and methods}

Tumor cell line. A2780, a widely used human ovarian cancer cell line (ATCC; Manassas, VA, USA) was maintained for 
5 days in Dulbecco's modified Eagle's medium (DMEM; Invitrogen; Thermo Fisher Scientific, Inc., Waltham, MA, USA) at $37^{\circ} \mathrm{C}$ in a humidified $5 \% \mathrm{CO}_{2}$ incubator. Medium supplements included $10 \%$ heat-inactivated fetal bovine serum and $1 \%$ penicillin-streptomycin (Gibco; Thermo Fisher Scientific, Inc.). Cells were grown to $70 \%$ confluence, then subjected to treatment with IL-33 or medium alone.

Treatment of ovarian carcinoma cells with IL-33. Once reaching $70 \%$ confluence, ovarian carcinoma cells were treated for 3 days with IL-33 (50 ng/ml; Shenandoah Biotechnology, Inc., Warwick, PA, USA) or medium alone at $37^{\circ} \mathrm{C}$. The concentration determined for experimental treatment was previously derived from our pilot experiments and previous cytokine studies (16-20).

Clonogenic survival assay. A total of 3 days post-incubation with IL-33, A2780 cells were detached and counted in a hemocytometer. A clonogenic survival assay was performed as described in our previous studies (16-18). The number of treatment colonies were then expressed as a percentage of total control colonies.

Determination of cell proliferation. Cell proliferation was quantified using the Quick Cell Proliferation Colorimetric Assay Kit (BioVision, Inc., Milpitas, CA, USA), which assesses for the degree of which cellular mitochondria cleave tetrazolium salts into formazan dye. The resulting concentration of formazan dye, detected at an absorbance of $440 \mathrm{~nm}$, is thus positively associated with the degree of mitochondrial dehydrogenase activity. Higher activity is positively associated with proliferation potential $(16,17,21,22)$.

Reverse transcription-polymerase chain reaction (RT-PCR). Experimental and control cells were washed with PBS and homogenized in TRIzol ${ }^{\circledR}$ (Invitrogen; Thermo Fisher Scientific, Inc.). RNA extraction was performed, and concentration was measured by NanoDrop (Thermo Fisher Scientific, Inc., Wilmington, DE, USA). RT-PCR was carried out using $1 \mu \mathrm{g}$ of A2780 mRNA, yielding cDNA aliquots proportional to mRNA expression profiles of our original cultures. Primer sequences specific for pro and anti-proliferative/apoptotic molecules, as well as for GAPDH were then amplified. GAPDH, a housekeeping gene, was used to internally control for differences in amounts of cDNA amplified. A more detailed description of our RT-PCR process, including semi-quantification methods and primer sequences, can be found in our previous cytokine studies (16-20).

Immunohistochemistry (IHC). Using a Cytopro cytocentrifuge (Wescor, Inc., Logan, UT, USA), A2780 ovarian cancer cells were spun into positively charged slides. IHC was used to stain for the proteins Fas cell surface death receptor (Fas) and p27. Cells were first incubated with $0.1 \%$ saponin in $1.0 \%$ BSA (Thermo Fisher Scientific, Inc.) for $30 \mathrm{~min}$ at room temperature, and then with the anti-Fas (cat. no. sc-716; Santa Cruz Biotechnology Inc.) and anti-p27 (cat. no. sc-528; Santa Cruz Biotechnology, Inc.) polyclonal antibodies for $60 \mathrm{~min}$ at $1 \mu \mathrm{g} / \mathrm{ml}$ at room temperature. Primary antibody incubation was followed by incubation with a biotinylated secondary antibody (711-065-152; Jackson ImmunoResearch Laboratories, Inc., West Grove, PA, USA) at $0.5 \mu \mathrm{g} / \mathrm{ml}$ for $30 \mathrm{~min}$. The avidin-biotin complex immunoperoxidase system (Vector Laboratories, Inc., Burlingame, CA, USA) was then used to detect immunoreactivity. Slides were developed using NovaRED (vector) as a chromogen. Hematoxylin was then applied as a counterstain for $30 \mathrm{~min}$ at room temperature. As a negative control, the primary antibody was replaced with an equal amount of rabbit IgG. Staining was observed under a Genco microscope (JC-311; magnification, x400; Jenco International Inc., Portland, OR) and MetaMorph version 6.3r6 (Molecular Devices, LLC, Sunnyvale, CA, USA) was used to measure the average intensity for p27 and Fas within the areas covered. Results are reported as an average staining intensity of 3 slides \pm the standard error of the mean relative to that in controls.

Caspase-3 activity. Caspase-3 activity was assayed using a caspase-3/CPP32 colorimetric assay kit (BioVision, Inc.), and increased activity of caspase-3 is positively associated with an increased propensity for apoptosis (16-20).

Statistical analysis. All experiments were performed in triplicate. Data are presented as the means + standard error of the means. Statistical analysis was conducted using an unpaired, two-tailed Student's t-test (Excel 2007; Microsoft Corporation, Redmond, WA, USA). $\mathrm{P}<0.05$ was considered to indicate a statistically significant difference.

\section{Results}

IL-33 favors proliferation and growth of ovarian cancer cells. When compared with controls, direct IL-33 $(50 \mathrm{ng} / \mathrm{ml})$ treatment in A2780 ovarian cancer cells induced a statistically significant increase in proliferation $(\mathrm{P}<0.05)$, as indicated by the results of the clonogenic survival assay (Fig. 1A) and the quick cell proliferation assay (Fig. 1B). These results clearly indicated that IL-33 has an oncogenic effect.

IL-33 downregulates the expression level of p27 of ovarian cancer cell. To investigate the direct effect of IL-33 on the expression of pro and anti-proliferative molecules, cell cultures of A2780 at $70 \%$ confluence were treated with IL-33 for 3 days at $50 \mathrm{ng} / \mathrm{ml}$. mRNA expression alteration for pro-proliferative molecules [Cyclins B, D, E, cyclin-dependent kinase (Cdk) 2 and Cdk4] and anti-proliferative molecules (p18, p21, p27, p53) was evaluated by RT-PCR. The results revealed a significant increase and a significant decease were in p21 and p27 expression levels, respectively, when compared with controls (Fig. 2). A decreased mRNA expression of p27 was further demonstrated by the IHC results (Fig. 3). A decreased expression of p27 induced by IL-33 may contribute to the effect of IL-33 on cell growth because p 27 is an anti-proliferative molecule in the cell cycle. An increased expression of p21 is unexpected and may be a compensatory effect.

IL-33 inhibits apoptosis of ovarian cancer cells. Apoptosis also contributes to the cellular population of ovarian cancer. To address if IL-33 has any effect on cellular apoptosis of A2780 ovarian cancer cells, a well-established apoptosis kit 

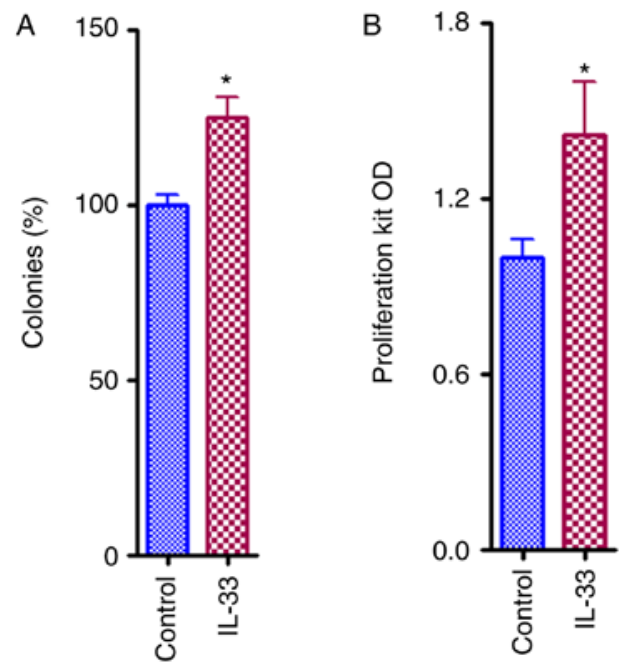

Figure 1. IL-33 favors proliferation and growth of A-2780 ovarian cancer cells. (A) Clonogenic survival assay results of A-2780 ovarian cancer cells treated with and without IL-33 $(50 \mathrm{ng} / \mathrm{ml})$. The number of colonies were counted and expressed as a percentage of total colonies in medium alone (control). (B) Proliferation kit results of A-2780 ovarian cancer cells treated with and without IL-33 $(50 \mathrm{ng} / \mathrm{ml})$. Results are expressed as the mean colony or OD values + standard error of the mean in both groups. ${ }^{*} \mathrm{P}<0.05$ vs. control. OD optical density; IL-33, interleukin-33.

was used. As presented in Fig. 4, the relative caspase-3 activity in IL-33 treated group decreased by $~ 50 \%$ compared with control group (Fig. 4; $\mathrm{P}<0.05$ ).
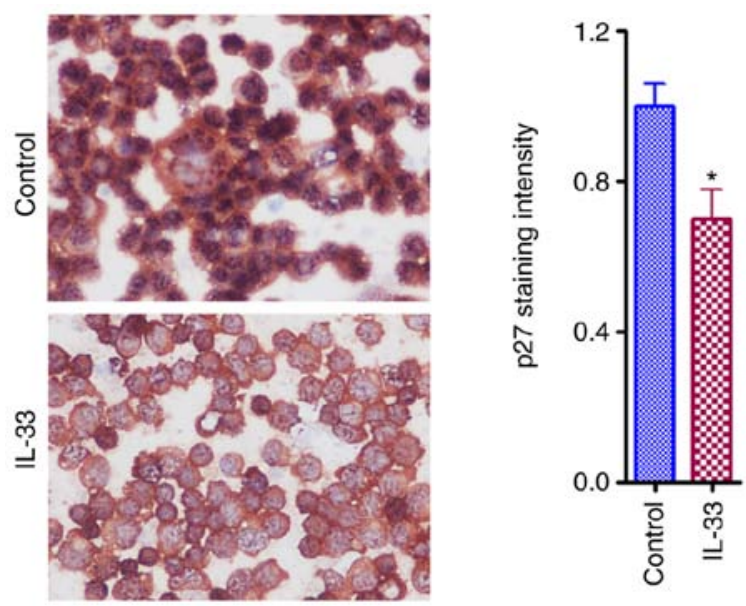

Figure 3. IL-33 downregulates the protein expression of p27 in ovarian cancer cells. Representative images of immunohistochemistry. Results are expressed as the mean staining intensity relative to that in control group + standard error of the mean in both groups. Original magnification, $x 400 .{ }^{*} \mathrm{P}<0.05$ vs. control. IL-33, interleukin-33.

IL-33 downregulates the expression levels of Fas and tumor necrosis factor-related apoptosis-inducing ligand receptor 1 (TRAILR1) in ovarian cancer cells. mRNA expression of pro-apoptotic molecules [Fas, Fas ligand, TRAIL, TRAILR1 and $\mathrm{B}$ cell lymphoma (Bcl)-2 associated $\mathrm{X}$, apoptosis regulator] as well as anti-apoptotic molecules (FLICE-inhibitory
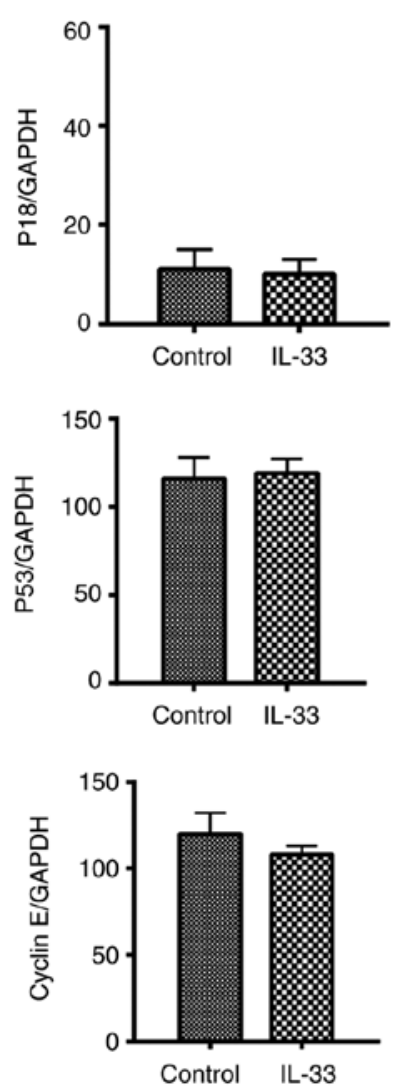
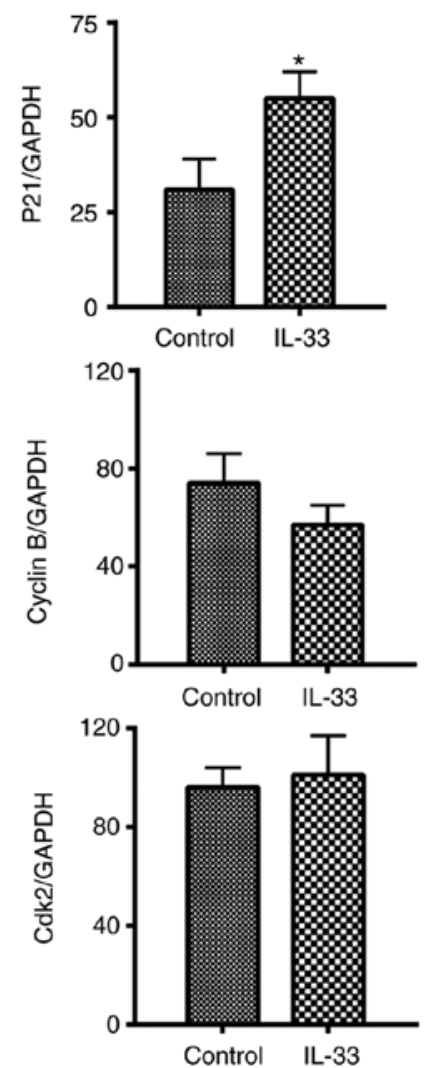
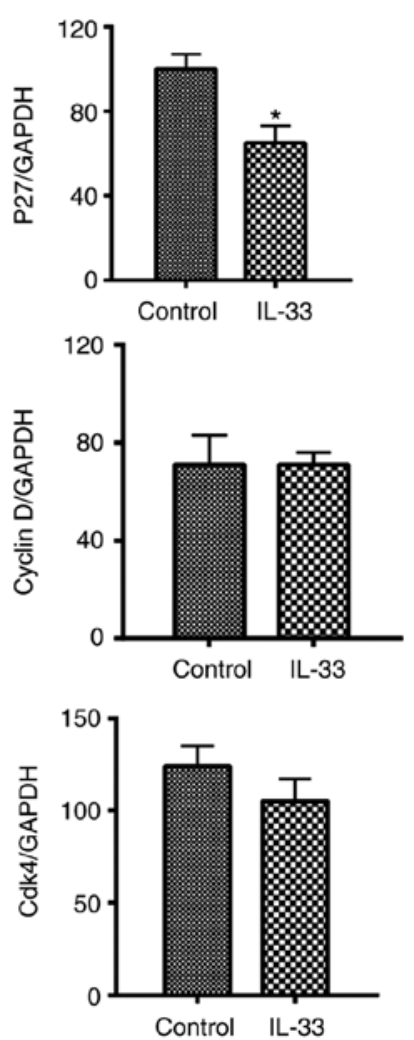

Figure 2. IL-33 downregulates the mRNA expression of p27 in ovarian cancer cells. mRNA expression levels of for pro-proliferative molecules (Cyclins B, $\mathrm{D}, \mathrm{E}, \mathrm{Cdk} 2$ and Cdk4) and anti-proliferative molecules (p18,p21, p27, p53), evaluated by reverse transcription-polymerase chain reaction. Results are expressed as the mean ratio of molecule densitometric Units/GAPDH + standard error of the mean (magnification, $\mathrm{x} 100)$. ${ }^{*} \mathrm{P}<0.05 \mathrm{vs}$. control. Cdk, cyclin-dependent kinase; IL-33, interleukin-33. 


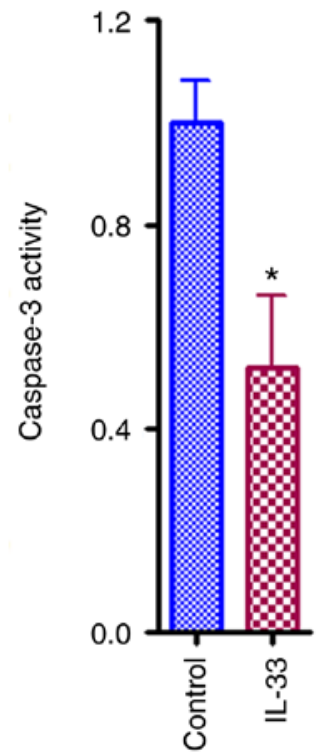

Figure 4. IL-33 inhibits apoptosis of A-2780 ovarian cancer cells. Caspase-3 activity is expressed as mean activity relative to control + standard error of the mean. ${ }^{*} \mathrm{P}<0.05$ vs. control. IL-33, interleukin-33.


Figure 5. IL-33 downregulates the mRNA expression levels of Fas and TRAILR1 in ovarian cancer cells. mRNA expression levels of pro-apoptotic molecules (Fas, FasL, TRAIL, TRAILR1 and Bax) as well as anti-apoptotic molecules (FLIP, Bcl-2, and Survivin) wereevaluated by reverse transcription-polymerase chain reaction. Results are expressed as the mean ratio of molecule densitometric Units/GAPDH + standard error of the mean (magnification, $\mathrm{x} 100$ ). ${ }^{*} \mathrm{P}<0.05$ vs. control. FasL, Fas ligand; TRAIL, tumor necrosis factor-related apoptosis-inducing ligand; TRAILR1, TRAIL receptor 1; Bcl-2, B cell lymphoma-2 Bax, Bcl-2 associated X, apoptosis regulator; FLIP, FLICE-inhibitory protein.
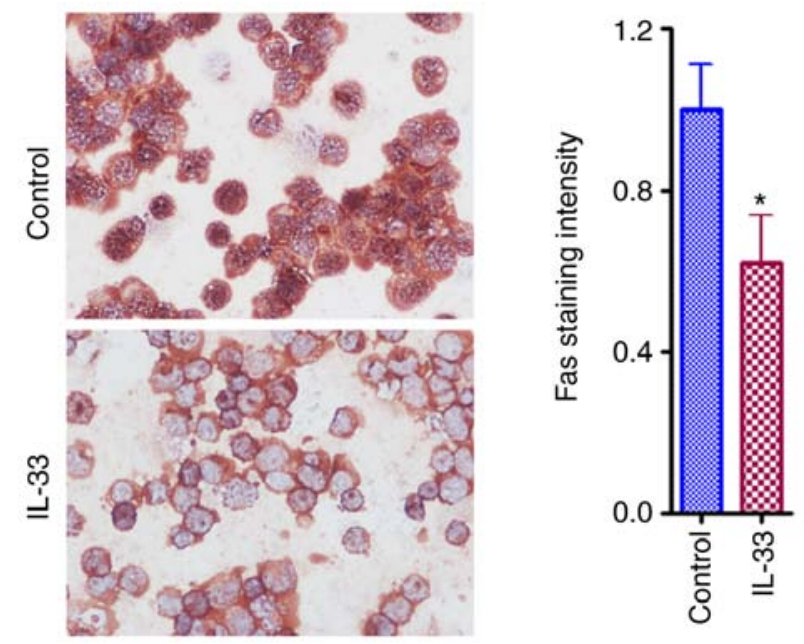

Figure 6. IL-33 downregulates the protein expression level of Fas in ovarian cancer cells. Representative images of immunohistochemistry. Results are expressed as the mean staining intensity relative to that in control group + standard error of the mean in both groups. Original magnification, $\mathrm{x} 400{ }^{*} \mathrm{P}<0.05$ vs. control. IL-33, interleukin-33.

protein, Bcl-2, and Survivin) were semi-quantified and compared between IL-33 treatment and control groups. It was demonstrated that mRNA levels of Fas and TRAILR1 significantly decreased, and levels of TRAIL significantly increased (Fig. 5; P<0.05). Consistent with decreased levels of mRNA detected by RT-PCR, IHC results for Fas protein levels were also significantly decreased (Fig. 6).

\section{Discussion}

Ovarian cancer is the most fatal gynecological malignancy and the fifth leading cause of cancer associated-mortality for women in the United States (23). Annually in the USA there are 22,000 newly diagnosed cases, and 14,000 associated fatalities (24). However, its pathogenesis still remains poorly understood $(25,26)$. A lack of knowledge of its pathogenesis and molecular mechanisms has made any endeavors into significantly reducing risk and mortality largely ineffective (27).

A report published in 2016 from the American Cancer Society highlights that if ovarian cancer is diagnosed while it is still inside the ovary (stages 1A or 1B) a 92\% 5-year survival rate can be expected-however, only $15 \%$ of all ovarian cancers are detected at this early stage (28). The remaining $85 \%$ of diagnoses are considerably less treatable, and they account for a severe descent in the 5-year survival rate; $45 \%$. Currently, ovarian cancer is treated upfront with surgery, followed by a chemotherapy regimen appropriate for its stage (28-30). However, with the further development and promise of immunomodulatory approaches to treatment, the mainstay will likely be guided towards more favorable remission rates, with less accompanying side effects throughout the treatment process (31). Of the possible pathways which may eventually be targeted in an immunologically orientated approach to treatment, the IL-33/ST2 axis provides initial promise. The present study demonstrated that IL-33 exhibited a pro-tumor effect by promoting growth and inhibiting apoptosis in ovarian cancer cells. The underlying potential 
molecular mechanisms may be due to downregulation of p27, Fas and TRAILR1. The study clearly indicated that the inhibition of IL-33 and its associated effects in the IL-33/ST2 signaling pathway may serve as a promising strategy to treat ovarian cancer. It is well-known that the IL-33/ST2 pathway activates $\mathrm{NF}-\kappa \mathrm{B}$ and mitogen-activated protein kinases (MAPK) $(8-10,15,32)$. This is the upstream of the effect of the IL-33/ST2 pathway which has been well studied. The present study focused on the downstream effects of the IL-33/ST2 pathway, including molecules directly associated with proliferation and apoptosis which are influenced by activation of the NF- $\kappa \mathrm{B}$ and MAPK.

Consistent with the results of the present study, previous studies in cholangiocarcinoma, colon cancer and breast cancer have all identified IL-33/ST2 signaling to be positively associated with tumor growth and metastasis (8-10). Interestingly, another study on ovarian cancer using an in vivo model found that IL-33 and ST2 are both highly upregulated in ovarian tumors, with an even higher level of expression in metastatic lesions (15). To further implicate IL-33 and ST2, this group also investigated and found expression of these two to be positively associated with extracellular signal-regulated kinase and c-Jun N-terminal kinase signaling pathways, both of which are known to promote metastasis $(15,32)$.

Of the known anti- and pro-proliferative molecules investigated in the present study, only p21 and p27 showed significant results, an increase and a decrease, respectively. p21 is a typical anti-proliferative molecule, a higher level of p21 does not favor cell growth and p21 may function as a negative regulator for cancer metastasis (33). Thus, it is possible that the increased level of p21 was a compensatory mechanism for ovarian cancer cells to avoid marked changes to their environment to keep the balance of pro- and anti-proliferative molecules, particularly when another anti-proliferative molecule p27 decreased. As a result, it may be possible that the downregulation of the anti-proliferative molecule p27 dominated and shifted the balance to favor cancer cell growth.

Decreased apoptosis is a well-known characteristic of cancer cells. In the present study, there was a notable decrease in apoptosis among ovarian cancer cells in the presence of IL-33, which suggested that IL-33 inhibited apoptosis of ovarian cancer cells. The intricacies of the molecular mechanism may be rather complex. Here, the expression of Fas and TRAILR1 was decreased in the presence of IL-33, which may elucidate the appearance of decreased apoptosis in the tissue, since both Fas and TRAILR1 are known pro-apoptotic molecules. Notably, another pro-apoptotic molecule TRAIL was unexpectedly upregulated in the presence of IL-33. This could also be due to a compensatory effort. When considering the alterations in p21 following the addition of IL-33, the seemingly paradoxical changes for these anti-proliferative and pro-apoptotic molecules indicated that it is not the change of one specific molecule, but the balance between pro- and anti-proliferative as well as pro- and anti-apoptotic molecules which determines the fate of ovarian cancer cells.

The pilot experiments from our lab indicated that IL-33 was not detected in A2780 ovarian cancer cells by immunohistostaining. We further checked online information regarding this issue our finding was consistent with the information in the RNA and protein expression database (http://www. proteinatlas.org/) which indicated that little RNA of IL-33 was detectable and IL-33 protein cannot be detected in $90 \%$ of ovarian cancer samples. Since no or little IL-33 is expressed in ovarian cancer cells, this may make knockdown of IL-33 unnecessary. If IL-33 was found highly/moderately expressed in a cancer cell line, then it would be critical to knock out or block endogenous IL-33 to further confirm our initial findings. Despite the fact that little or no IL-33 is expressed on ovarian cancer cells, exogenous IL-33 from stroma cells of ovarian cancer could possibly be the source of IL-33 that promotes growth of ovarian cancer (34).

In conclusion, A2780 ovarian cancer cells undergo pro-proliferative and anti-apoptotic changes in the presence of IL-33, which lead to increased culture number and proliferative potential. The downregulation of p27 as well as Fas and TRAILR1 is very likely to be involved in these changes. The present study suggests that IL-33 may play a role in the promotion of ovarian cancer tumorigenesis, and consequently, may be a therapeutic target for better management of ovarian cancer.

\section{Acknowledgements}

The authors would like to thank Miss Mikayla M. Brockmeyer (Des Moines University) and Mr. Dylan Weir (University of Missouri) for their constructive comments regarding the revision of this manuscript.

\section{Funding}

The present study was supported by Des Moines University (grant no. IOER 112-3749 to YF).

\section{Availability of data and materials}

Data and materials are available upon request.

\section{Authors' contributions}

YF conceived and designed the study. XL, DMH, NJT, ZZ, AA, CQ, QB, XC and YF performed the experiments. YF, XL, DMH, NJT, ZZ, GW, MBN and MRW analyzed and interpreted the data. XL, NJT and YF wrote the draft. YF, MBN, MRW, GW, AA, CQ, QB and XC made critical revisions. All authors read and approved the final version of the manuscript.

\section{Ethical approval and consent to participate}

Ethical approval was obtained from Des Moines University and the University of Missouri IRB for the use of human cell lines.

\section{Patient consent for publication}

Not applicable.

\section{Competing interests}

The authors declare that they have no competing interests. 


\section{References}

1. Lataifeh I, Marsden DE, Robertson G, Gebski V and Hacker NF: Presenting symptoms of epithelial ovarian cancer. Aust N Z J Obstet Gynaecol 45: 211-214, 2005.

2. Vine MF, Calingaert B, Berchuck A and Schildkraut JM: Characterization of prediagnostic symptoms among primary epithelial ovarian cancer cases and controls. Gynecol Oncol 90: 75-82, 2003.

3. Orsulic S, Li Y, Soslow RA, Vitale-Cross LA, Gutkind JS and Varmus HE: Induction of ovarian cancer by defined multiple genetic changes in a mouse model system. Cancer Cell 1: 53-62, 2002.

4. Moss HA, Berchuck A, Neely ML, Myers ER and Havrilesky LJ: Estimating cost-effectiveness of a multimodal ovarian cancer screening program in the United States: Secondary analysis of the UK collaborative trial of ovarian cancer screening (UKCTOCS). JAMA Oncol 4: 190-195, 2018.

5. Sporn MB: The war on cancer. Lancet 347: 1377-1381, 1996.

6. Schmitz J, Owyang A, Oldham E, Song Y, Murphy E, McClanahan TK, Zurawski G, Moshrefi M, Qin J, Li X, et al: IL-33, an interleukin-1-like cytokine that signals via the IL-1 receptor-related protein ST2 and induces T helper type 2-associated cytokines. Immunity 23: 479-490, 2005.

7. Sanada S, Hakuno D, Higgins LJ, Schreiter ER, McKenzie AN and Lee RT: IL-33 and ST2 comprise a critical biomechanically induced and cardioprotective signaling system. J Clin Invest 117: $1538-1549,2007$.

8. Li J, Razumilava N, Gores GJ, Walters S, Mizuochi T, Mourya R, Bessho K, Wang YH, Glaser SS, Shivakumar P and Bezerra JA: Biliary repair and carcinogenesis are mediated by IL-33-dependent cholangiocyte proliferation. J Clin Invest 124: 3241-3251, 2014.

9. Liu X, Zhu L, Lu X, Bian H, Wu X, Yang W and Qin Q: IL-33/ST2 pathway contributes to metastasis of human colorectal cancer. Biochem Biophys Res Commun 453: 486-492, 2014.

10. Jovanovic IP, Pejnovic NN, Radosavljevic GD, Pantic JM Milovanovic MZ, Arsenijevic NN and Lukic ML: Interleukin-33/ ST2 axis promotes breast cancer growth and metastases by facilitating intratumoral accumulation of immunosuppressive and innate lymphoid cells. Int J Cancer 134: 1669-1682, 2014.

11. Hu LA, Fu Y, Zhang DN and Zhang J: Serum IL-33 as a diagnostic and prognostic marker in non-small cell lung cancer Asian Pac J Cancer Prev 14: 2563-2566, 2013.

12. Gao X, Wang X, Yang Q, Zhao X, Wen W, Li G, Lu J, Qin W, Qi Y, Xie F, et al: Tumoral expression of IL-33 inhibits tumor growth and modifies the tumor microenvironment through $\mathrm{CD}^{+}$ T and NK cells. J Immunol 194: 438-445, 2015.

13. Feng W, Shi P, Lei B, Chai X and Tan C: IL-33 influenced the development of colorectal cancer via regulating Fra-1. Int J Clin Exp Pathol 10: 467-472, 2017.

14. Fang Y, Zhao L, Xiao H, Cook KM, Bai Q, Herrick EJ, Chen X, Qin C, Zhu Z, Wakefield MR, et al: IL-33 acts as a foe to MIA PaCa-2 pancreatic cancer. Med Oncol 34: 23, 2017.

15. Tong X, Barbour M, Hou K, Gao C, Cao S, Zheng J, Zhao Y, $\mathrm{Mu} \mathrm{R}$ and Jiang HR: Interleukin-33 predicts poor prognosis and promotes ovarian cancer cell growth and metastasis through regulating ERK and JNK signaling pathways. Mol Oncol 10: 113-125, 2016.

16. Fang Y, DeMarco VG and Nicholl MB: Resveratrol enhances radiation sensitivity in prostate cancer by inhibiting cell proliferation and promoting cell senescence and apoptosis. Cancer Sci 103: 1090-1098, 2012.
17. Fang Y, Herrick EJ and Nicholl MB: A possible role for perforin and granzyme $\mathrm{B}$ in resveratrol enhanced radiosensitivity of prostate cancer. J Androl 33: 752-760, 2012.

18. Fang Y, Chen X, Bai Q, Qin C, Mohamud AO, Zhu Z, Ball TW, Ruth CM, Newcomer DR and Herrick EJ: IL-9 inhibits HTB-72 melanoma cell growth through upregulation of p21 and TRAIL. J Surg Oncol 111: 969-974, 2015.

19. Fang Y, Sharp GC, Yagita H and Braley-Mullen H: A critical role for TRAIL in resolution of granulomatous experimental autoimmune thyroiditis. J Pathol 216: 505-513, 2008.

20. Fang Y, Wei Y, Demarco V, Chen K, Sharp GC and BraleyMullen H: Murine FLIP transgene expressed on thyroid epithelial cells promotes resolution of granulomatous experimental autoimmune thyroiditis in DBA/1 mice. Am J Pathol 170: 875-887, 2007.

21. Fang Y, Bradley MJ, Cook KM, Herrick EJ and Nicholl MB: A potential role for resveratrol as a radiation sensitizer for melanoma treatment. J Surg Res 183: 645-653, 2013.

22. Fang Y,Bradley MJ, Bai Q, Cook KM,Herrick EJ and Nicholl MB: Hydrogen peroxide enhances radiation-induced apoptosis and inhibition of melanoma cell proliferation. Anticancer Res 33: 1799-1807, 2013.

23. Brucks JA: Ovarian cancer. The most lethal gynecologic malignancy. Nurs Clin North Am 27: 835-845, 1992.

24. Siegel RL, Miller KD and Jemal A: Cancer statistics, 2016. CA Cancer J Clin 66: 7-30, 2016.

25. Daniilidis A and Karagiannis V: Epithelial ovarian cancer. Risk factors, screening and the role of prophylactic oophorectomy. Hippokratia 11: 63-66, 2007.

26. Landen CN Jr, Birrer MJ and Sood AK: Early events in the pathogenesis of epithelial ovarian cancer. J Clin Oncol 26: 995-1005, 2008.

27. Kurman RJ and Shih IeM: The origin and pathogenesis of epithelial ovarian cancer-a proposed unifying theory. Am J Surg Pathol 34: 433-443, 2010.

28. Roett MA and Evans P: Ovarian cancer: An overview. Am Fam Physician 80: 609-616, 2009.

29. Lalrinpuii E, Bhageerathy PS, Sebastian A, Jeyaseelan L, VinothaThomas, Thomas A, Chandy R and Peedicayil A: Ovarian cancer in young women. Indian J Surg Oncol 8: 540-547, 2017.

30. Pignata S, Cannella L, Leopardo D, Pisano C, Bruni GS and Facchini G: Chemotherapy in epithelial ovarian cancer. Cancer Lett 303: 73-83, 2011.

31. Patil US, Jaydeokar AV and Bandawane DD: Immunomodulators: A pharmacological review. Int J Pharm Pharm Sci 4 (Suppl 1): S30-S36, 2012.

32. Fu H, Hu Z, Wen J, Wang K and Liu Y: TGF-beta promotes invasion and metastasis of gastric cancer cells by increasing fascin1 expression via ERK and JNK signal pathways. Acta Biochim Biophys Sin 41: 648-656, 2009.

33. Deryabin PI, Borodkina AV, Nikolsky NN and Burova EB: Relationship between $\mathrm{p} 53 / \mathrm{p} 21 / \mathrm{Rb}$ and MAPK signaling pathways in human endometrium-derived stem cells under oxidative stress. Tsitologiia 57: 788-795, 2015

34. Chen X, Lu K, Timko NJ, Weir DM, Zhu Z, Qin C, Mann JD, Bai Q, Xiao H, Nicholl MB, et al: IL-33 notably inhibits the growth of colon cancer cells. Oncol Lett 16: 769-774, 2018. 